ANN. ZOOTECH.

\title{
MAMMITE STAPHYLOCOCGIQUE DE LA BREBIS INFLUENCE DES MODES DE TRAITE ET DE SEVRAGE, DU NOMBRE D'AGNEAUX, DU STADE DE LACTATION ET DE LA PRODUCTION LAITIËRE SUR LE DECCLENCHEMENT DE L'INFECTION
}

PAR

\section{PLOMMET et G. RICORDEAU}

Station Centrale de Microbiologie et Recherches Laitières, Station de Recherches sur l'Élevage, C. N. R. Z., Jouy-en-Josas (S. et O.),

\section{SOMMAIRE}

Les auteurs ont étudié dans deux troupeaux de brebis laitières l'évolution de deux épizooties de mammite staphylococcique. Ils ont observé la répartition des cas de mammite dans des lots de brebis soumis à des conditions d'élevage différentes et discuté du mécanisme susceptible d'expliquer les différences de sensibilité observées.

A 1'occasion d'expériences sur la lactatio:1 de la brebis, nous avons pu observer, pendant deux années, de nombreux cas de mammites staphylococciques sur les troupeaux du Centre National de Recherches Zootechniques, particulièrement sur les animaux de certains lots soumis à des conditions expérimentales bien définies. Un certain nombre de facteurs sont apparus comme susceptibles, à priori, de favoriser l'apparition des mammites. Nous avons retenu dans notre étude les modes de traite et de sevrage, le nombre d'agneaux allaités, le stade de lactation et la production laitière des brebis.

\section{I. - CONDITIONS EXPÁRIMENTALES}

Les troupeaux comprennent essentiellement des brebis laitières de race Préalpes. Ces brebis sont logées dans deux bergeries (1'une située à Jouy-en-Josas, l'autre à la ferme de Brouessy) et réparties en lots. Ces 
lots, dont les caractéristiques sont données dans le tableau I, se distinguent d'après le nombre d'agneaux à la naissance et les conditions de sevrage et de traite. Le groupe "divers » comprend d'une part des brebis qui ont été retirées des lots en expérience à la suite d'une mammite apparue pendant l'allaitement, d'autre part des brebis d'un lot en réserve et atteintes de mammite pendant la lactation.

\section{A. - Nombre d'agneaux.}

En I957, le troupeau comprenait go mères dont 79 simples (brebis ayant I agneau) et II doubles, ou " bessonnières " (brebis ayant 2 agneaux). En I958, I97 mères dont 43 doubles. Au total, 287 lactations ont été étudiées.

$$
\text { B. 一 Traite. }
$$

En règle générale la traite est faite deux fois par jour soit à la main, soit à la machine; dans ce cas, on a utilisé une machine Alfa-Laval à Brouessy, une machine Gascoigne à Jouy-en-Josas. Deux types de manchons-trayeurs ont été utilisés en r958 sur la machine Alfa-Laval. Dans les deux bergeries, la traite mécanique est suivie d'un égouttage à la main avec sous-battage appelé " repasse ». Les quantités de lait récoltées lors de la traite mécanique et de la repasse ont été enregistrées quotidiennement.

Seules les brebis du lot 6 , soumises à la traite mécanique après sevrage r rutal, ont été traites 3 fois par jour, pendant les deux premières semaines de traite.

\section{C. - Sevrage.}

Les agneaux ont été sevrés soit le $5^{\mathrm{e}}$, soit le $2 \mathrm{I}^{\mathrm{e}}$, soit enfin le $42^{\mathrm{e}}$ jour après l'agnelage. Deux méthodes de sevrage ont été appliquées : le sevrage brutal et le sevrage progressif. Dans le prenier cas, on passe de l'allaitement à la traite sans transition. Dans le second cas, les brebis allaitent leurs agneaux pendant la nuit (de I $8 \mathrm{~h}$ à $6 \mathrm{~h}$ ) et sont traites une seule fois par jour pendant les I5 jours précédant le sevrage complet.

\section{D. - CARACTÉRISATION DES MAMMITES.}

Les mammites ont été reconnues cliniquement, puis confirmées par examen bactćriologique dans tous les lots en I957 et dans les lots I, 2, 3, 4, 5 en I958. De plus, en I958, les lots $6,7,8,9$, Io ont été soumis à des contrôles bactériologiques périodiques systématiques permettant de déceler les infections latentes. Toutes les infections staphylococciques reconrues étaient des $\mathrm{i}$ fectiors cliniques généralement aiguës ou surai- 
(9, I960) MAMMITE STAPHYLOCOCCIQUE DE LA BREBIS

\section{TABl ${ }_{4}$ AUU I}

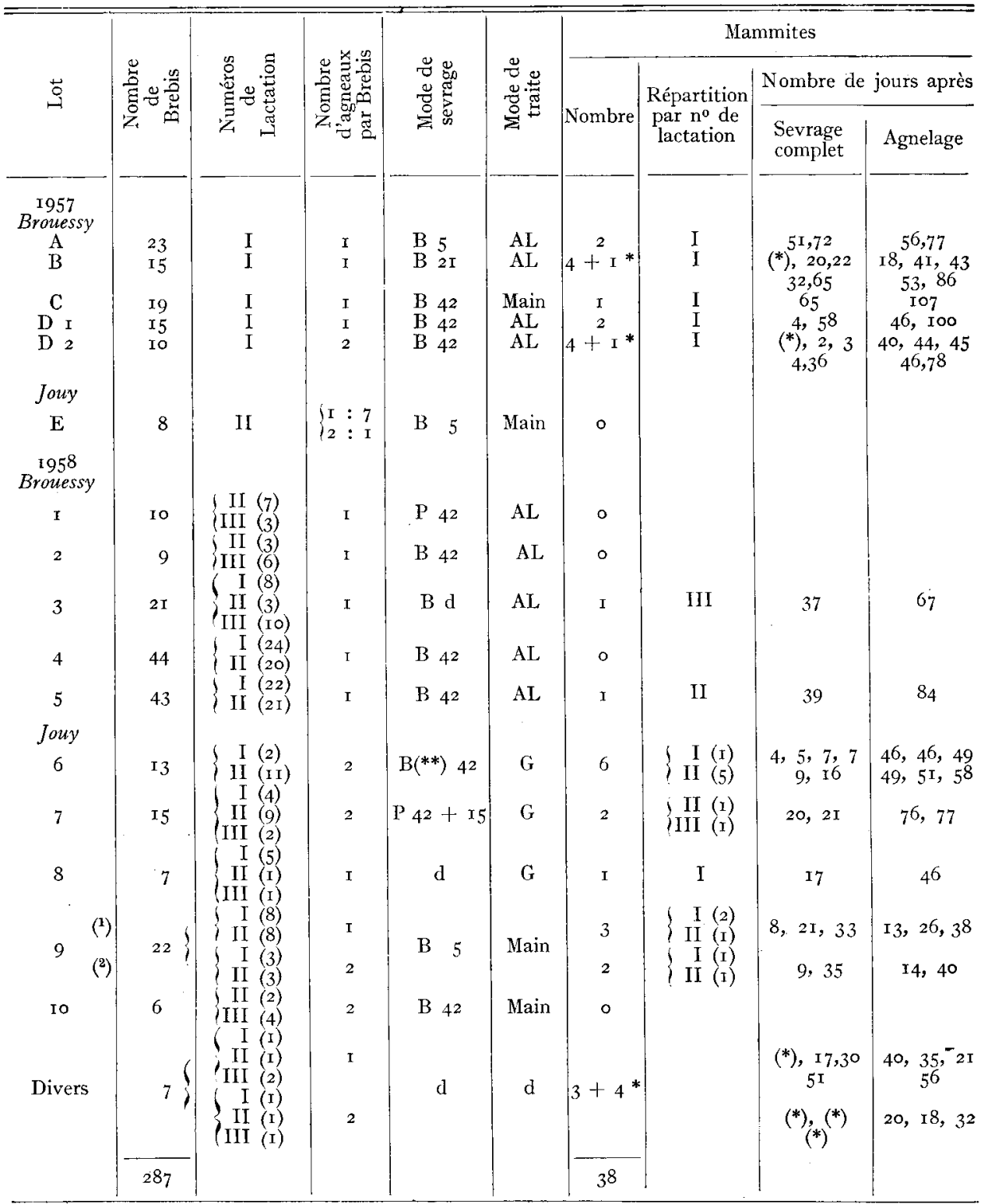

Constitution des lots en expérience

Numéros de laclation : I, II $=$ brebis en $\mathrm{I}^{\mathrm{re}}$ ou $2^{\mathrm{e}}$ lactation $;$ III $\Rightarrow$ brebis en $3^{\mathrm{e}}, 4^{\mathrm{e}}$ ou $5^{\mathrm{e}}$ lactation.

Sevrage : B $42=$ brutal, après 42 jours d'allaitement. $\mathrm{P}_{42}+\mathrm{I}_{5}=$ progressif, d'une durée de $\mathrm{I} 5$ jours, après 42 jours d'allaitement. $\mathrm{B}\left({ }^{* *}\right)=$ sevrage brutal suivi de trois traites par jour pendant $\mathrm{I} 5$ jours. Mode de traite : $\mathrm{AL}=$ machine Alfa Laval; $\mathrm{G}=$ machine Gascoigne $; \mathrm{d}=$ divers modes de sevrage et de traite dans le lot. Mammites $:(*)=$ indique une mammite apparue pendant l'allaitement. 
guës (I seul cas de mammite chronique, décelable facilement par ailleurs). Dans ces conditions, aucune mammite staphylococcique n'a pu échapper à l'observation, même en l'absence de contrôle bactériologique systématique.

A côté des infections staphylococciques, on a décelé des infections imputables à différents germes et qui se présentaient soit sous une forme aiguë, soit sous une forme chronique apparente ou non. Ces infections n'ont pas été incluses dans la présente étude.

\section{E. - CONTRÔLES BACTÉRIOLOGIQUES.}

I es contrôles systématiques ont eu lieu environ une fois par mois; et, au moment du sevrage, dans la plupart des lots, une fois toutes les 3 semaines. En cas de mammite, dans tous les lots, plusieurs contrôles successifs sont pratiqués avant et après traitement. Le prélèvement des échantillons de lait est fait avant la traite, après désinfection des trayons par l'alcool à $70^{\circ}$. Les échantillons sont ensemencés, dans l'heure qui suit le prélèvement ou après une nuit au réfrigérateur : on étale $0,025 \mathrm{ml}$ de lait sur une demi-boite de gélose au sang de mouton. Quelques échantillons ont, en outre, été incubés à $37^{\circ} \mathrm{C}$ pendant $\mathrm{I} 8$ heures avant un deuxième ensemencement. Tous les résultats positifs ont été confirmés par mesure de la réaction cellulaire, soit par numération des cellules totales (selon la technique de BREED avec le colorant de Newman), soit par le test au Teepol de SCHalm et Noorlander (I957).

I es germes pathogènes rencontrés ont été repiqués puis identifiés; les staphylocoques, en particulier, ont fait l'objet d'une étude spéciale qui sera publiée ultérieurement. La plupart des sonches de staphylocoques sont du type toxinique $\alpha \beta$; la souche isolée du cas unique de mammite chronique observé à l'agnelage est du type $\beta$ pur. Bien que la toxine $\delta$ n'ait pas été recherchée systématiquement, il semble que la plupart des souches produisaient cette toxine. Toutes les souches isolées en 1958 ont été typées par la méthode des phages $\left.{ }^{1}{ }^{1}\right)$ : sur 24 souches, 2 I appartenaient au même groupe phagique caractérisé par les phages : $88, \mathrm{I} 74 / \mathrm{I} 6,42 \mathrm{D}$, $42 \mathrm{E}$; les 3 autres souches appartenaient à divers groupes.

\section{I. - Caractères clinigues et Évolution des mammites.}

Sauf dans le cas déjà signalé, toutes les mammites staphylococciques observées sont des infections à départ soudain et à évolution très rapide. En effet, 4 brebis ont été contrôlées respectivement le $2^{\mathrm{e}}, 3^{\mathrm{e}}, 5^{\mathrm{e}}$ et $7^{\mathbf{e}}$ jours précédant l'apparition des signes cliniques : aucune n'éliminait de germes au moment de ce contrôle. La multiplication bactérienne précède

(I) Nous remercions le Dr WaIl, de l'Institut Pasteur de Paris, qui a bien voulu se charger de cette identification. 
donc immédiatement l'infection clinique. L'évolution clinique est toujours rapide ; il s'écoule moins de $48 \mathrm{~h}$ avant que la lésion définitive s'établisse. Dans un cas extrême, une brebis qui avait donné à la traite du matin une production quantitativement normale a été trouvée morte d'une mammite gangréneuse dans l'après-midi. Dans la plupart des cas, malgré la rapidité du traitement (antibiotiques et corticoïdes administrés par voie générale et locale) l'évolution vers une lésion irréversible est à peu près constante. Le bilan de I958 est le suivant : gangrène et mort : I ; gangrène : 2 ; forme grave évoluant vers l'hypertrophie et l'abcédation : I5; stabilisation, passage à l'état chronique avec foyer induré et infection persistante, lactation arrêtée : I ; même type de lésion mais lactation maintenue : 3 ; guérison clinique et bactériologique : 2 . (Au total, 24 cas, une brebis ayant été atteinte successivement dans les 2 glandes.)

\section{II. - RÉSULTATS}

Comme toutes les maladies infectieuses, les mammites sont le résultat, d'une part de l'action pathogène d'un germe microbien, d'autre part de la résistance de 1'organisme à cette action pathogène. Il faut donc, pour étudier les variations de résistance ou de sensibilité de l'organisme à l'infection, se placer dans des conditions telles que l'action pathogène soit sensiblement identique sur tous les animaux. L'action pathogène est elle-même fonction d'une part de la virulence propre à chaque souche microbienne, d'autre part de la contagion, c'est-à-dire des risques pour l'organisme de se trouver en contact avec l'agent pathogène. Pour que les résultats - exprimés en pourcentage d'animaux infectés dans chaque lot - soient strictement comparables, il faudrait que tous les animaux soient soumis au même risque de contagion par tue souche microbienne de pouvoir pathogène constant.

Dans la présente étude portant sur deux années successives et deux bergeries différentes, toutes les souches de staphylocoques isolées étaient du même type toxinique ; de plus, la deuxième année, dans les deux bergeries, les souches appartenant aı même type phagique avaient vraisemblablement la même origine. On peut donc admettre, sans risque d'erreur grave, que le pouvoir pathogène de la souche contaminante n'a pas sensiblement varié au cours de l'observation.

D'autre part, si on étudie !a marche de l'épizootje dans les deux bergeries, au cours des deux années successives, on s'aperçoit que dans deux cas (bergerie de Brouessy, I957 ; bergerie de Jouy, 1958) cette marche a été comparable : les mammites ont commencé à apparaitre dès la période d'allaitement et ont continué à se produire pendant les deux premiers mois de la lactation. Dans les deux autres cas, au contraire, ou bien 
il n'y a pas en de mammites (Jouy, I957) ou bien celles-ci sont apparues tardivement (Brouessy, I958) à un moment, comme nous le verrons plus loin, où la plupart des animaux du troupeau pouvaient être considérés comme n'étant plus sensibles à la contagion. Si on aj̣oute à cette observation le fait que dans les lots comparables des deux bergeries (Brouessy, I957 ; et Jouy, r958), par exemple les lots D2 et 6 , les pourcentages de cas de mammites sont voisins, on peut admettre que les conditions de contagion étaient sinon identiques du moins très voisines dans les deux troupeaux au cours de ces deux lactations. Par contre, les conditions de contagion à Jouy en I957 et à Brouessy en I 958 étaient manifestement très différentes.

Pour cette raison, dans tous les résultats qui suivent, nous n'avons pas tenu compte des lots E, I, 2, 3, 4 et 5. Par contre, nous avons fait l'hypothèse que toutes les brebis des lots $A, B, C, I), 6,7,8,9$, Io et " divers " étaient soumises à la même contagion par wne souche de staphylocoque douée d'un pouvoir pathogène constant.

\section{A. - STADE DE SEVRAGE.}

A Brouessy en I957, nous avons comparé le sevrage précoce, semiprécoce et tardif sur trois lots de brebis allaitant un seul agneau et soumises à la traite mécanique (tableau II).

\section{Tableau II}

Influence du stade de sevrage.

Brebis allaitant 1 agneau el mises à la traile mécanique.

\begin{tabular}{c|c|c|c}
\hline \hline \multirow{2}{*}{$\begin{array}{c}\text { Nombre de jours après } \\
\text { agnelage }\end{array}$} & Nombre de brebis & \% de inammite \\
\cline { 2 - 3 } & sans mammite & avec mammite & \\
\cline { 2 - 3 } 5 & 2r & 2 & 8,7 \\
$2 \mathrm{I}$ & Io & 4 & 28,6 \\
42 & r3 & 2 & 13,3 \\
\hline
\end{tabular}

Le test d'indépendance $\left(\chi^{2}\right.$ d'homogénéité - en appliquant la correction de continuité de YATES) ne révèle aucune différence siguificative entre les lots $(\mathrm{P}>\mathrm{O}$, IO). On peut donc pour les brebis avec un seul agneau, tenir le stade de sevrage comme un facteur sans influence nette sur l'apparition des mammites.

\section{B. - Mode de traite.}

Bien que deux types de trayeuses aient été utilisés et pour l'une d'elles deux modèles de manchons, il n'a pas été possible d'établir de distinction entre ces machines, ni entre les manchons, le nombre d'animaux 
ou le nombre de cas de mammites dans chacun des lots étant trop petit pour permettre de déceler une différence significative. Par exemple dans les deux lots de doubles mises à la traite mécanique après sevrage brutal (lots D2 et 6) le pourcentage de mammites fut très élevé avec l'un et 1'autre type de trayeuses.

TABLEAU III

Influence du mode de traite.

\begin{tabular}{|c|c|c|c|}
\hline & \multicolumn{2}{|c|}{ Nombre de brebis } & \multirow{2}{*}{$\%$ de mammite } \\
\hline & Sans mammite & Avec mammite & \\
\hline $\begin{array}{l}\text { Traite mécanique : } \\
\quad \text { I agneau } \ldots \ldots \ldots \ldots \\
\quad 2 \text { agneaux } \ldots \ldots \ldots\end{array}$ & $\begin{array}{l}44 \\
\mathrm{I} 2\end{array}$ & $\begin{array}{r}8 \\
10\end{array}$ & $\begin{array}{l}15,4 \\
45,4\end{array}$ \\
\hline $\begin{array}{l}\text { Traite manuelle : } \\
\quad \text { I agneau } \ldots \ldots \\
2 \text { agneaux ....... }\end{array}$ & $\begin{array}{l}3 \mathrm{I} \\
\mathrm{IO}\end{array}$ & $\begin{array}{l}4 \\
2\end{array}$ & $\begin{array}{l}10,2 \\
16,6\end{array}$ \\
\hline
\end{tabular}

Comparaison de la traite mécanique et de traite manuelle après servage brutal pour les brebis ayant allaité 1 ou 2 agneaux.

On peut essayer d'autre part de comparer la traite à la machine à la traite manuelle. Les résultats obtenus (tableau III) ne font apparaître aucun effet significatif du mode de traite lorsqu'on considère séparément les brebis avec I ou 2 agneaux $(P \geqslant 0, I 9$ - calcul direct des fréquences). En combinant les données relatives aux deux catégories de brebis la proportion des mammites dans le groupe trait à la machine, pourrait-être considérée, à la limite, comme significativement supérieure à celle observée dans le groupe soumis à la traite manuelle (24,3 p. Ioo contre I2,7 p. IOo). Si la probabilité d'obtenir les proportions observées est faible (o,xo $\left.>\frac{\mathrm{P}}{2}>0,05\right)$, on ne peut cependant rien conclure, compte-tenu d'une part du petit nombre d'observation, d'autre part de la proportion légèrement différente des brebis avec I oul 2 agneaux, suivant le mode de traite.

En effet, c'est surtout entre ces deux catégories de brebis qu'apparaissent nettement des différences de sensibilité : ainsi, pour le même mode de traite mécanique (tableau. III), les doubles manifestent une sensibilité très supérieure à celle des brebis ayant allaité un seui agneaul $\left(\frac{\mathrm{P}}{2}<\mathrm{o}, \mathrm{OI}\right)$.

\section{C. - MODE DE SEVRAGE.}

I,e mode de sevrage des agneaux - brutal ou progressif - est susceptible de conditionner l'adaptation des brebis à la traite mécanique : 
on peut donc penser qu'il favorise plus ou moins l'éclosion de mammites lors du passage de l'allaitement à la traite.

TABLEAU IV

Influence du mode de serrage des agneaux sur la fréquence d'apparition des mammites lors de la phase de traite complète.

\begin{tabular}{l|c|c|c|c}
\hline \hline & Sevrage (1) & Nombre de brebis & $\begin{array}{c}\% \\
\% \\
\text { de mammite }\end{array}$ \\
\hline Brebis doubles .... & $\begin{array}{c}\text { Brutal } \\
\text { Progressif }\end{array}$ & $\begin{array}{c}\text { Sans mammite } \\
\text { Avec mammite }\end{array}$ & $\begin{array}{c}45,4 \\
13\end{array}$ \\
\hline Brebis simples .... & Brutal & I3 & 2 & I3,3 \\
\hline
\end{tabular}

(1) Sevrage 42 jours après l'agnelage ; traite mécanique.

Dans le tableau IV, nous avons comparé les résultats dans les lots soumis soit au sevrage bruta1, scit au sevrage progressif, en considérant toujours les deux catégories de brebis (simples et doubles). Pour les doubles, le sevrage progressif est plus favorable que le sevrage brutal $\left(0,05>\frac{P}{2}>0,01\right)$. Ce résultat est encore plus significatif si l'on note, d'une part que la comparaison des deux modes de sevrage a lieu en partie dans la même bergerie et presque simultanément, d’autre part que les deux cas de mammite du lot soumis au sevrage progressif ne sont apparus que 20 et 2 I jours après le sevrage complet, c'est-à-dire assez loin des influences du sevrage sur la production, alors que 8 mammites sur Io sont apparues au cours de la première semaine chez les brebis soumises au sevrage brutal. Toutefois, si l'on tient compte du lot de doubles soumises à la traite manuelle après sevrage brutal ( 2 mammites sur I2 brebis), la fréquence des mammites n'est plus que de 35,3 p. Ioo et l'effet du mode de sevrage n'est plus significatif $(\mathrm{P}>0,20)$.

En ce qui concerne les brebis avec un agneau, nous n'avons pas de réstultats valables, mais par comparaison avec les chiffres obtenus sur les doubles, on peut constater que le sevrage brutal n'a pratiquement pas d'influence. I,es rares infections enregistrées par ailleurs dans lots I, 2, 3, 4 et 5 confirment cette conclusion.

\section{I). - Nombre D'agniatix ad Sevrage.}

Comme l'indiquent les résultats précédents, les brebis ayant allaité deux agneaux sont beaucoup plus sensibles que les autres.

Nous avons indiqué dans le tableau $V$, la répartition globale des mammites en fonction du nombre d'agneaux, de la bergerie et de l'année. 
Le pourcentage moyen des mammites est de 16,5 p. Ioo pour les brebis avec un agneau contre $28,6 \mathrm{p}$. Ioo pour les doubles. Cette différence de susceptibilité, que nous avions déjà enregistrée dans le cas de la traite mécanique succédant à un sevrage brutal, se retrouve de façon significative $\left(0,10>\frac{P}{2}>0,05\right)$ pour l'ensemble des animaux soumis aux différents modes de sevrage et de traite.

TABLEAU V

Influence du nombre d'agneaux au sevrage.

\begin{tabular}{|c|c|c|c|}
\hline & \multicolumn{2}{|c|}{ Nombre de brebis } & \multirow{2}{*}{ de mammite } \\
\hline & Sans mammite & Avec mammite & \\
\hline $\begin{array}{l}\text { Brebis simples } \\
\text { Jouy I } 958 \ldots \ldots \ldots \ldots \ldots \ldots \\
\text { Brouessy I } 957 \ldots \ldots \ldots \ldots \ldots\end{array}$ & $\begin{array}{l}19 \\
62\end{array}$ & $\begin{array}{l}6 \\
9\end{array}$ & $\left.\begin{array}{l}26,0 \\
12,7\end{array}\right\}$ moyenne 16,5 \\
\hline $\begin{array}{l}\text { Brebis doubles } \\
\quad \text { Jouy } 195^{8} \ldots \ldots \ldots \ldots \ldots \\
\text { Brouessy r } 957 \ldots \ldots \ldots \ldots \ldots\end{array}$ & $\begin{array}{r}30 \\
5\end{array}$ & $\begin{array}{r}\text { IO } \\
4\end{array}$ & \begin{tabular}{l|l}
25,0 & moyenne 28,6 \\
44,4 & mone
\end{tabular} \\
\hline
\end{tabular}

Ajoutons également que, sur la totalité des lots considérés uniquement pendant la période d'allaitement, c'est-à-dire en dehors des influences du sevrage et de la traite, on a observé deux cas de mammites chez 60 brebis simples (3,3 p. I00) et 4 cas chez 47 doubles $(8,5$ p. I00).

\section{E. - STADE DE IACTATION.}

I a dernière colonne du tableau I indique le nombre de jours séparant l'agnelage du moment de 1'apparition des mammites. Ce nombre varie de $I_{3}$ à ro 7 jours pour des durées moyennes de lactation de l'ordre de 150 jours. Il en ressort que $76 \mathrm{p}$. Ioo des mammites se déclarent avant le $6 \mathrm{o}^{\mathrm{e}}$ jour de lactation et $95 \mathrm{p}$. Ioo avant le $9 \mathrm{o}^{\mathrm{e}}$ jour.

Dans la figure I et le tableau VI, ncus avons schématisé, pour chaque catégorie de mères ( $\mathrm{I}$ ou 2 agneaux) la fréquence d'apparition des mammites à partir du début de la traite complète. Il apparaît très nettement que dans le groupe de brebis ayant allaité un agneau les mammites se déclarent d'une manière assez uniforme au cours des ro semaines qui suivent le sevrage, alors que dans le groupe des doubles les mammites apparaissent très rapidement après le sevrage : en $2 \mathrm{I}$ jours, $86 \mathrm{p}$. Ioo des mammites de ce groupe se sont en effet déclarées.

Cette différence dans la répartition dans le temps des mammites est à rapprocher de l'influence du mode de sevrage; dans les lots D2 et 6 (sevrage brutal suivi de traite à la machine) la plupart des mammites 
se déclarent dans les jours qui suivent le sevrage, alors que dans les lots 7 et 9 l'apparition des mammites est plus tardive.

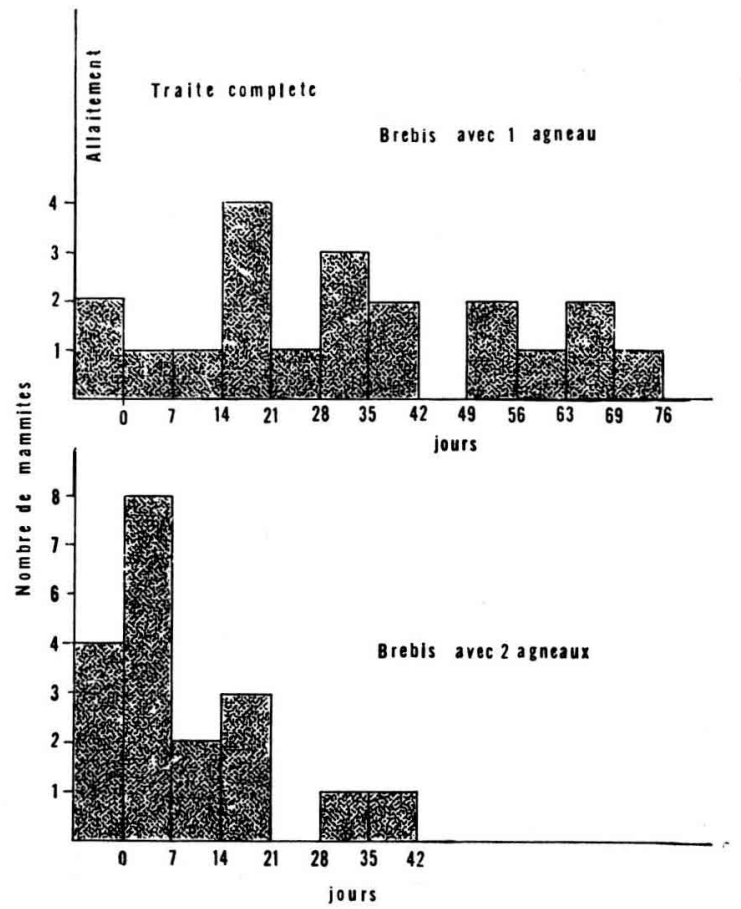

Fig. I. - Répartition des cas de mammites au cours de la période de traite.

TABLEAl VI
Répartition des cas de mammites
dans les semaines qui suivent la mise à la traite.

\begin{tabular}{|c|c|c|c|c|}
\hline \multirow{2}{*}{$\begin{array}{l}\text { Nombre de semaines après } \\
\text { la mise à la traite }\end{array}$} & \multicolumn{2}{|c|}{$\begin{array}{c}\text { Nombre total de mammites } \\
\text { déclarées }\end{array}$} & \multicolumn{2}{|c|}{$\begin{array}{l}\text { Pourcent des mammites totales } \\
\text { de chaque groupe }\end{array}$} \\
\hline & Simples & Doubles & Simples & Doubles \\
\hline Pendant l'allaitenent..... & 2 & 4 & Io & 22 \\
\hline I. $\ldots \ldots \ldots \ldots \ldots \ldots$ & 3 & I I & I5 & $6 \mathbf{I}$ \\
\hline $2 \ldots \ldots \ldots \ldots \ldots \ldots \ldots$ & 4 & 13 & 20 & $7^{2}$ \\
\hline $3 \ldots \ldots \ldots \ldots \ldots \ldots \ldots$ & 8 & I6 & 40 & 80 \\
\hline $4 \ldots \ldots \ldots \ldots \ldots \ldots$ & 9 & 16 & 45 & 89 \\
\hline $5 \ldots \ldots \ldots \ldots \ldots \ldots \ldots$ & 12 & I 7 & 60 & 95 \\
\hline $6, \ldots \ldots \ldots \ldots \ldots \ldots \ldots$ & 14 & 18 & 70 & 100 \\
\hline $\bar{\imath} \ldots \ldots \ldots \ldots \ldots \ldots \ldots$ & I4 & & 70 & \\
\hline $8 \ldots \ldots \ldots \ldots \ldots \ldots \ldots$ & 96 & & 80 & \\
\hline $9 \ldots \ldots \ldots \ldots \ldots \ldots \ldots$ & 17 & & 85 & \\
\hline 10. & I9 & & 95 & \\
\hline II $\ldots \ldots \ldots \ldots \ldots \ldots \ldots$ & 20 & & 100 & \\
\hline
\end{tabular}


F. - INFLUENCE DU NIVEAU DE PRODUCTION ET DE L'IMPORTANCE

DE LA " REPASSE ".

A l'intérieur de chaque lot, les facteurs susceptibles de modifier la production laitière (alimentation, durée de la lactation, nombre d'agneaux) sont, en règle générale, homogènes. Dans ces conditions, la production d'une des brebis à l'intérieur d'un lot est une valeur qui peut être comparée valablement à la production moyenne du lot. On peut ainsi classer une brebis comme bonne, moyenne ou faible laitière. Si l'on admet que dans chaque lot les brebis les plus sensibles aux infections de la mamelle sont celles qui ont effectivement fait une mammite, il devient possible d'établir une relation entre la production laitière des brebis et leur prédisposition aux mamnites.

\section{I. - Production totale.}

Ia production laitière réelle s'exprime par un volume de lait produit au cours d'une lactation. Nous avons considéré le cas des brebis atteintes de mammites en I958 et nous avons comparé, dans le tableau VII, leurs productions individuelles de 1957 aux productions moyennes des lots correspondants.

\section{TABLEAU VII}

Production laitière totale en 1957 des brebis atteintes de mammite en 1958, rapportée à la production movenne totale des brebis du lot correspondant

\begin{tabular}{|c|c|c|c|c|c|}
\hline Brebis $n^{0}$ & $\begin{array}{l}\text { Nombre de brebis } \\
\text { dans le lot ayant } \\
\text { servi au calcul } \\
\text { de la moyenne }\end{array}$ & $\begin{array}{l}\text { Nombre de } \\
\text { jours } \\
\text { de traite }\end{array}$ & $\begin{array}{c}\text { Production totale } \\
\text { moyenne } \\
\text { du lot (litres) }\end{array}$ & $\begin{array}{c}\text { Production totale } \\
\text { de la brebis } \\
\text { (litres) }\end{array}$ & $\begin{array}{l}\text { Production de la } \\
\text { brebis en } \% \text { de } \\
\text { la moyenne } \\
\text { du lot }\end{array}$ \\
\hline $\mathbf{I} \ldots \ldots$ & 9 & $150-160$ & I.37 & I 28 & 93 \\
\hline $2 \ldots \ldots \ldots$ & I3 & I05 & $5^{2}$ & $4 \mathrm{r}$ & 79 \\
\hline $3 \ldots \ldots$ & I 3 & 105 & 52 & 53 & 102 \\
\hline $4 \ldots \ldots \ldots$ & 8 & I $52-160$ & I I 2 & 74 & 66 \\
\hline $5 \ldots \ldots \ldots$ & I I & 117 & 99 & I $2 \mathrm{I}$ & I 22 \\
\hline $6 \ldots \ldots \ldots$ & $I_{7}$ & 103 & 77 & 74 & 96 \\
\hline $7 \ldots \ldots \ldots$ & 17 & 103 & 77 & 77 & 100 \\
\hline & I 7 & 103 & 77 & 90 & I I 7 \\
\hline $9 \ldots \ldots \ldots$ & I4 & 95 & 73 & $5 \mathrm{I}$ & 70 \\
\hline Moyenne .. & & & & & 94 \\
\hline
\end{tabular}

Ia production de ces brebis ne s'écarte pas significativement de la moyenne. Il est cependant difficile de tirer des conclusions rigoureuses: d'une part, le nombre d'animaux contrôlés sur la lactatio: antérieure est faible, d'autre part, la corrélation entre deux lactations successives n'est pas très étrcite; aussi la mesure de la production de la lactation antérieure ne représente-t-elle pas avec certitude la valeur potentielle de la lactation en cours. 


\section{II. - Production au moment de l'infection.}

Par ailleurs, puisque les mammites n'apparaissent que pendant les premiers mois de la lactation, le niveau de production au début de la lactation, et plus précisément, au moment même où se déclare l'infection, est une valeur plus intéressante à considérer que la production totale, hypothétique d'ailleurs, de la lactation en cours.

C'est pourquoi nous avons comparé la production des brebis au moment où se déclare l'infection, à la production moyenne des brebis du lot correspondant au même stade de traite (tableau VIII). La production de la brebis est, en principe, estimée par la moyenne des 7 jours de traite précédant la mammite; si la mammite apparaît avant le $7^{\mathrm{e}}$ jour de traite,

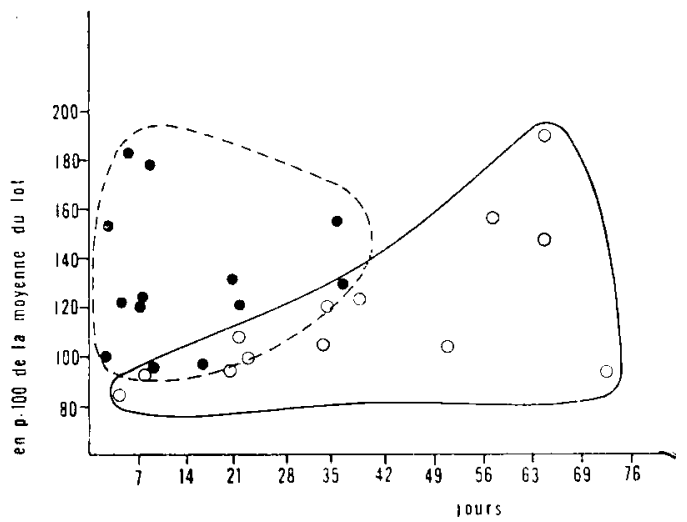

FIG. 2. - Distribution des cas de mammites dans le temps en fonction de la production des brebis. P'roduction exprimée en pourcent de la moyeme du lot correspondant au même stade de traite.

.Brebis avec un agneau : point blanc. Brebis avec deux agneaux : point noir.

la moyenne est calculée sur la totalité des jours de traite. La production moyenne du lot est établie par semaine, au même stade de traite, sur l'ensemble des brebis constituant le lot restant en expérience à la fin de lactation. I es résultats sont exprimés pour chaque brebis en p. Ioo de la production moyenne du lot. La même comparaison est établie pour la " repasse " journalière des brebis traites à la machine. I,es comparaisons figurent dans le tableau VIII, sauf celles relatives aux lots 3 , 8 et "divers " représentant des groupes hétérogènes; elles ont été établies séparément pour les simples et pour les doubles, et dans l'ordre d'apparition des mammites après la mise à la traite.

Ces résultats que nous avons rapportés dans la figure 2 sont assez nets : au moment du déclenchement de l'infection, la production laitière des brebis avec un ou deux agneaux est égale respectivement à II7 et I3I p. Ioo de la production moyenne des autres brebis.

Cependant le faible effectif des lots et l'échelonnement des mammites 


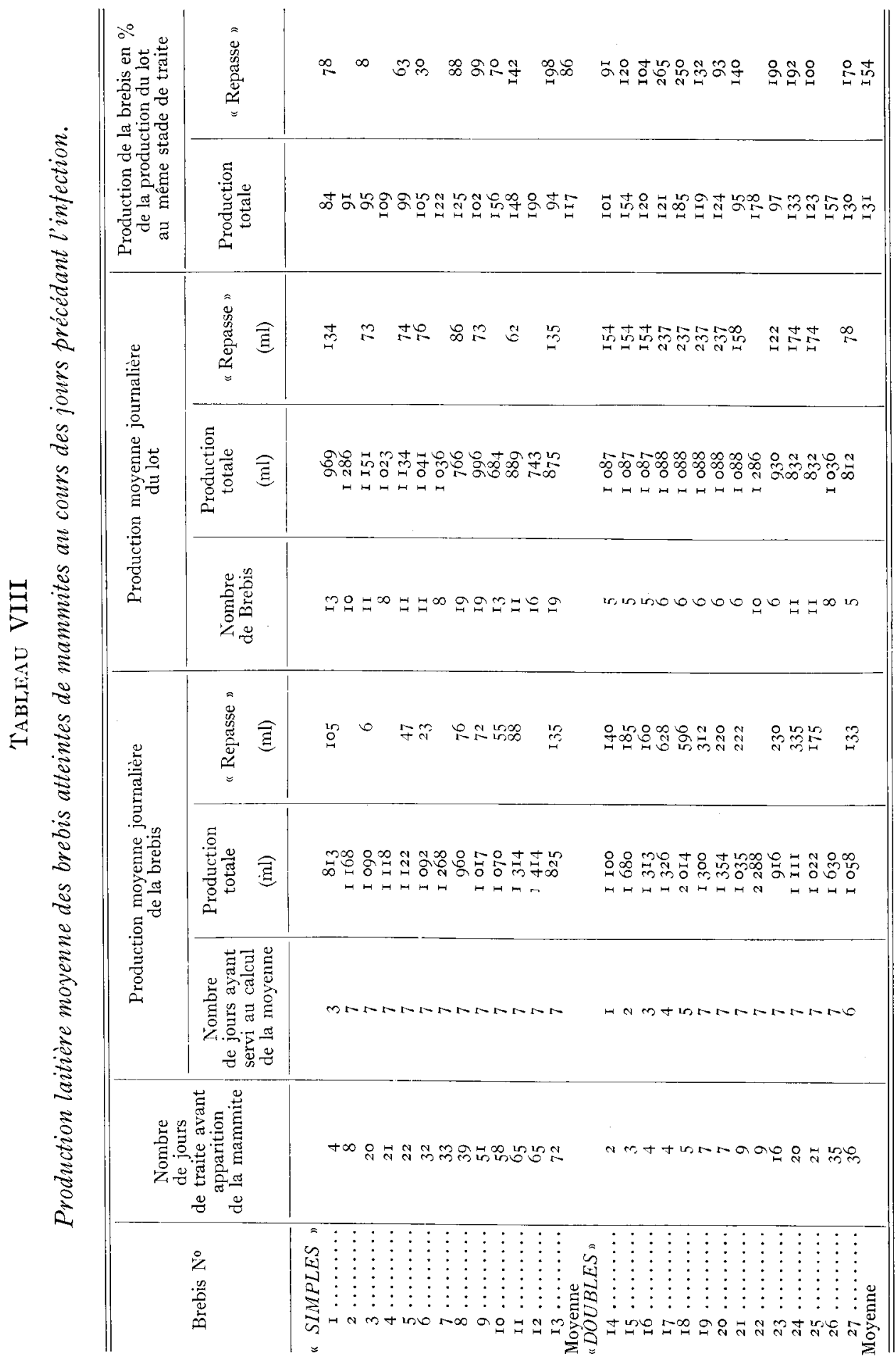


dans le temps ne permet pas d'utiliser des tests précis pour l'interprétation de ces résultats. On peut cependant dire que dans l'ensemble ce sont les brebis les plus productrices qui sont atteintes.

$$
\text { III. - "Repasse ». }
$$

Les variations individuelles et journalières des quantités de lait recueillies à la repasse sont telles que l'on observe des écarts importants dans les moyennes des lots et que la "repasse " de chaque brebis exprimée en $p$. roo de la moyenne accuse de larges écarts. Il est cependant remarquable de constater que la "repasse " des simples est inférieure à la moyenne (86 p. Ioo), alors que celle des doubles est très supérieure à la moyenne (I54 p. IOO).

\section{III. - DISCUSSION}

Il est bien certain qu'il existe d'autres facteurs de prédisposition aux mammites que ceux étudiés ici : l'entraînement du personnel à la traite et le réglage de la machine à traire sont probablement les plus importants. Ces facteurs étant sensiblement équivalents dans tous les lots ne modifient pas les résultats en ajoutant leur effet à ceux des facteurs étudiés.

Nous n'avons pas tenu compte de l'âge des brebis parce que la plu-

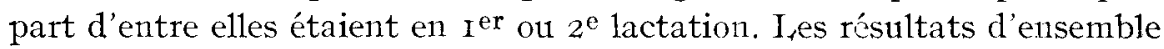
concernant la comparaison des productions laitières des brebis en fonction du numéro de lactation ne font d'ailleurs apparaître à cet égard aucune différence appréciable. Quoi qu'il en soit, la répartition par âge indiquée au tableau I montre qu'en I958, dans la bergerie de Jouy, les mammites ont atteint aussi bien les animaux en première lactation ( 7 cas, 29 p. IOO), en deuxième lactation (Io cas, $27 \mathrm{p}$. IOo) ou au-delà ( 4 cas, 40 p. IOO). Ceci est contraire à l'opinion fréquemment admise selon laquelle la sensibilité des brebis diminuerait avec 1'âge.

Ises résultats obtenus par nous ne portent que sur un petit nombre d'animaux; ils ne permettent pas d'étudier avec certitude l'influence de facteurs secondaires. Ils ont cependant mis en évidence l'influence indiscutable I $^{\circ}$ ) du stade de lactation (début de la période de traite qui est aussi période de production maximum) ; $2^{\circ}$ ) du niveau de production; $\left.3^{\circ}\right)$ du nombre d'agneaux; $4^{\circ}$ ) du sevrage brutal, dans le cas de traite mécanique, sur les brebis doubles.

Parmi ces facteurs les trois premiers cits agissent probablement de la même manière, et sont en relation avec la quantité totale de lait sécrétée. Ricordeau, Denamur et Martinet (I960) ont montré qu'il existe une relation étroite entre la quantité de lait cobtenue à la traite et le débit 
de lait enregistré au cours des 15 premières secondes de traite : l'écoulement de lait des meilleures brebis est donc plus intense au début de la traite, ce qui traduit certainement des différences dans le diamètre du sphincter du trayon ou du volume citernal. On sait que chez la vache il existe une relation directe entre la taille de 1'orifice du trayon et le débit de lait maximum (BAXTER, ClARKE, Dodd et FooT, r950) et une autre relation directe entre le débit de lait et la sensibilité aux infections de la mamelle (DODD et NEAVE, I95I). On explique cette dernière relation par le fait que la pénétration des microorganismes dans la mamelle est d'autant plus facile que 1'orifice du trayon est plus large. La même hypothèse appliquée à la brebis donnerait l'explication des résultats que nous avons observés.

Le sevrage brutal, suivi de traite mécanique, a peut-être un mode d'action différent. L'étude de la consommation du lait par les agneaux a permis à 1'un de nous (G. R.) en collaboration avec Denamur (I960) de montrer que le passage brutal de l'allaitement à la traite se traduit par une baisse de la production, de l'ordre de 33 p. Ioo. En valeur absolue, cette différence est plus élevée pour les doubles que pour les simples puisque ces dernières ont une production laitière au cours de l'allaitement très inférieure à celle des doubles. Celles-ci sont tétées par deux agneaux à la fois avec une fréquence au moins aussi grande pour chacun des agneaux que dans le cas des simples. La baisse de production au moment du sevrage correspond donc à un phénomène de rétention, qui se traduit en particulier chez les doubles par une quantité de lait assez élevée à la repasse. I1 est vraisemblable que cette rétention est extrêmement favorable à la multiplication des germes pathogènes dans la mamelle et offre ainsi aux staphylocoques éventuellement présents un maximum de chances de réussir à infecter la mamelle.

\section{SUMMARY}

In two flocks of milking ewes, the time of appearance and the distribution in different groups (table I) of 34 cases of staphylococcal mastitis was studied for two successive years (in 287 lactations). Hand or machine milking (table III) has no significant effect on the occurrence of mastitis except in ewes with twins when they are weaned abruptly. Furthermore, abrupt weaning (table IV) with machine milking has a significant effect on ewes with twins but not on ewes with singles. The influence of the number (table V) of lambs is significant for the ewes as a whole but it appears to be connected particularly with abrupt weaning and machine milking.

Mastitis occurs especially at the start of the milking period, in the first three months for the ewes with singles, and in the first three weeks for those with twins (table VI).

Finally, it is the best milkers, on average, which are affected (table VIII). 
The mechanism whereby milking ewes are predisposed to mastitis under these various influences is discussed.

\section{RÉFÉRENCES BIBLIOGRAPHIQUES}

Baster E. S., Clarke P. M., Dodd F. H., Foot A. S., I950. Factors affecting the rate of machine milking. J. Dairy res., 17, II7-I27.

DoDD F. H., NEAVE F. K., I95I. Machine milking rate and mastitis. J. Dairy res., 18, I99-204.

Ricordeau G., Denamur R., Martinet J., Ig6o. Résultats non publiés.

Schal, O. V., NOORLaNder D. O., I957. Experiments and observations leading to development of the California Mastitis Test. J. Amer. Vet. Med. Ass., 130, I99-204. 